\title{
Molecular cloud formation and the star formation efficiency in M 33
}

\section{Molecule and star formation in M 33}

\author{
J. Braine ${ }^{1}$, P. Gratier ${ }^{1}$, C. Kramer ${ }^{2}$, K. F. Schuster ${ }^{3}$, F. Tabatabaei ${ }^{4}$, and E. Gardan ${ }^{1}$ \\ ${ }^{1}$ Laboratoire d'Astrophysique de Bordeaux, Université de Bordeaux, OASU, CNRS/INSU, 33271 Floirac, France \\ e-mail: braine@obs.u-bordeaux1.fr \\ 2 IRAM, Avenida Divina Pastora, 7, Nucleo Central, 18012 Granada, Spain \\ IRAM, 300 Rue de la piscine, 38406 St Martin d'Hères, France \\ 4 Max-Planck Institut für Radioastronomie, Auf dem Hügel 69, 53121 Bonn, Germany
}

Received 31 January 2010 / Accepted 2 July 2010

ABSTRACT

\begin{abstract}
Does star formation proceed in the same way in large spirals such as the Milky Way and in smaller chemically younger galaxies? Earlier work suggests a more rapid transformation of $\mathrm{H}_{2}$ into stars in these objects but (1) a doubt remains about the validity of the $\mathrm{H}_{2}$ mass estimates and (2) there is currently no explanation for why star formation should be more efficient. M 33, a local group spiral with a mass $\sim 10 \%$ and a metallicity half that of the Galaxy, represents a first step towards the metal poor Dwarf Galaxies. We have searched for molecular clouds in the outer disk of M 33 and present here a set of detections of both ${ }^{12} \mathrm{CO}$ and ${ }^{13} \mathrm{CO}$, including the only detections (for both lines) beyond the $R_{25}$ radius in a subsolar metallicity galaxy. The spatial resolution enables mass estimates for the clouds and thus a measure of the $N\left(\mathrm{H}_{2}\right) / I_{\mathrm{CO}}$ ratio, which in turn enables a more reliable calculation of the $\mathrm{H}_{2}$ mass. Our estimate for the outer disk of M 33 is $N\left(\mathrm{H}_{2}\right) / I_{\mathrm{CO}(1-0)} \sim 5 \times 10^{20} \mathrm{~cm}^{-2} /\left(\mathrm{K} \mathrm{km} \mathrm{s}^{-1}\right)$ with an estimated uncertainty of a factor $\leq 2$. While the ${ }^{12 / 13} \mathrm{CO}$ line ratios do not provide a reliable measure of $N\left(\mathrm{H}_{2}\right) / I_{\mathrm{CO}}$, the values we find are slightly greater than Galactic and corroborate a somewhat higher $N\left(\mathrm{H}_{2}\right) / I_{\mathrm{CO}}$ value. Comparing the $\mathrm{CO}$ observations with other tracers of the interstellar medium, no reliable means of predicting where $\mathrm{CO}$ would be detected was identified. In particular, $\mathrm{CO}$ detections were often not directly on local HI or FIR or $\mathrm{H} \alpha$ peaks, although generally in regions with FIR emission and high HI column density. The results presented here provide support for the quicker transformation of $\mathrm{H}_{2}$ into stars in $\mathrm{M} 33$ than in large local universe spirals.
\end{abstract}

Key words. galaxies: groups: individual: M 33 - Local Group - galaxies: evolution - galaxies: ISM - ISM: clouds - stars: formation

\section{Introduction}

Several recent papers (Leroy et al. 2006; Gardan et al. 2007; Gratier et al. 2010b) have suggested that the rate of transformation of molecular gas $\left(\mathrm{H}_{2}\right)$ into stars is higher in small chemically young galaxies - those with low metallicities. The latter articles devoted much attention to whether the conversion factor from $\mathrm{CO}$ to $\mathrm{H}_{2}, N\left(\mathrm{H}_{2}\right) / I_{\mathrm{CO}}$, usually expressed in $\mathrm{H}_{2}$ molecules $\mathrm{cm}^{-2} /\left(\mathrm{K} \mathrm{km} \mathrm{s}^{-1}\right)$, could be severely underestimated. In this article, we present sensitive measurements of the $\mathrm{CO}$ emission from the outer disk of M 33, allowing us to estimate Virial masses for isolated molecular clouds far from the center as well as some ${ }^{13} \mathrm{CO}$ line measurements, providing a further check on the physical conditions in the Giant Molecular Clouds (GMCs) in M 33. A further goal is naturally to understand the mechanisms of molecular cloud formation and the outer disk provides a means to explore physical conditions unexplored by earlier work which only presented molecular cloud data in the inner disk where the stellar mass surface density dominates that of the gas.

In particular, we present data on a series of mid to outer disk clouds in M 33, including an interarm GMC with no measured associated star formation (H $\alpha$ or Far-IR emission) and a GMC beyond the $R_{25}$ radius. Because the stellar population in M 33 is rather young, the $R_{25}$ radius (30.8' Paturel et al. 2003), defined in B band, corresponds to an extremely low stellar surface mass density, well below that of the gas at the same radius. In M 33, the radius at which stellar and gaseous surface densities are equal is about $0.5 R_{25}$ whereas in large spirals the stars dominate until about the $R_{25}$ radius.

It has long been known that the amount of $\mathrm{CO}$ emission per unit star formation or per unit $\mathrm{H}_{2}$ is lower, sometimes much lower, in galaxies with subsolar metallicity (e.g. Rubio et al. 1991). In the solar neighborhood, the conversion factor $N\left(\mathrm{H}_{2}\right) / I_{\mathrm{CO}}$ is of order $2 \times 10^{20} \mathrm{~cm}^{-2} /\left(\mathrm{K} \mathrm{km} \mathrm{s}^{-1}\right)$ with a strong increase from the center to the outer disk (e.g. Sodroski et al. 1995). This radial variation is not only true for the Galaxy but also for other spirals where it has been studied (Braine et al. $1997 \mathrm{a}, \mathrm{b})$. The amount of $\mathrm{CO}$ emission per $\mathrm{H}_{2}$ mass varies not only with metallicity but also with the radiation field, which can act to raise the $\mathrm{CO}$ emission by warming the gas. If the metallicity is low the effect is opposite because the lower dust content and lesser degree of self-shielding by molecules other than $\mathrm{H}_{2}$ reduce the size of the CO-emitting regions with respect to the $\mathrm{H}_{2}$ cloud size, such that the $\mathrm{CO}$ emission principally traces the central regions of molecular clouds. Because the CO lines are optically thick, moderate changes in metallicity and radiation field with respect to Galactic conditions (solar vicinity or molecular ring) can be expected to only generate moderate changes in the $N\left(\mathrm{H}_{2}\right) / I_{\mathrm{CO}}$ ratio. 
The standard techniques to estimate the $\mathrm{H}_{2}$ mass, and thus $N\left(\mathrm{H}_{2}\right) / I_{\mathrm{CO}}$ ratio, are through the dust emission and an assumed dust-to-gas mass ratio, the "virial" mass from the cloud size and line width, and from optically thin tracers such as ${ }^{13} \mathrm{CO}$. We discuss the latter two methods in Sects. 4.1 and 4.2. The resulting $N\left(\mathrm{H}_{2}\right) / I_{\mathrm{CO}}$ is then used to estimate the star formation efficiency (SFE), defined as the ratio of the star formation rate per unit $\mathrm{H}_{2}$, in M 33.

M 33 has a metallicity a factor 2 below solar (Rosolowsky \& Simon 2008; Magrini et al. 2009), a moderate UV radiation field, and is a spiral galaxy despite having a mass $\sim 10$ times lower than the Galaxy. It thus represents a first step towards the still smaller, lower metallicity, irregular galaxies in the Local Group and beyond. Based on the above, we assume an Oxygen abundance of $12+\log (\mathrm{O} / \mathrm{H})=8.4-0.03 R_{\mathrm{kpc}}$. The distance to $\mathrm{M} 33$ is assumed to be $840 \mathrm{kpc}$ and we adopt an inclination angle of $56^{\circ}$ and a position angle of $22.5^{\circ}$ as in Gardan et al. (2007).

\section{Observations}

The observations presented here are a follow-up to the Gardan et al. (2007) mapping of a large part of M 33. All data were taken with the $30 \mathrm{~m}$ telescope run by the Institut de RadioAstronomie Millimétrique (IRAM) on Pico Veleta near Granada, Spain. Observing runs were in April, August, and Nov. of 2006.

The ${ }^{12} \mathrm{CO}$ and ${ }^{13} \mathrm{CO} J=1 \rightarrow 0$ and $J=2 \rightarrow 1$ transitions were observed with the "AB" receivers. These receivers are dual polarization and the beam-splitter enables simultaneous observation of the two transitions. Data were taken under good conditions, with system temperatures $\left(T_{\mathrm{a}}^{*}\right)$ of $300-400 \mathrm{~K}$ except for the ${ }^{13} \mathrm{CO}(1-0)$ where the average system temperature was $\sim 200 \mathrm{~K}$. As a result, data reduction was simple: bad channels were eliminated, a zero-order baseline (constant value) was subtracted, and spectra were averaged for each position.

The positions observed are shown as yellow and black circles in Fig. 1 for the ${ }^{12} \mathrm{CO}$ and red or blue circles for the ${ }^{13} \mathrm{CO}$. The size illustrates the IRAM CO(1-0) half-power beamsize. Generally, both the $J=1 \rightarrow 0$ and $J=2 \rightarrow 1$ transitions were observed simultaneously. The data are presented using the main beam temperature scale. The main beam efficiencies are estimated to be $0.72,0.74,0.53$, and 0.54 for respectively the lines at 115, 110, 230, and $220 \mathrm{GHz}$ (see http://www . iram. es/IRAMES/mainWiki/Iram30mEfficiencies) and forward efficiencies $0.95,0.95,0.91$, and 0.91 .

Table 1 provides the positions at which we observed ${ }^{12} \mathrm{CO}$. Tables 2 and 3 give the ${ }^{12} \mathrm{CO} J=1 \rightarrow 0$ and $J=2 \rightarrow 1$ fluxes at each detected position and the rms noise level at $1 \mathrm{MHz}$ resolution for the undetected positions. The results of fitting Gaussian line profiles to the spectra, in order to robustly measure line widths and velocities, can be found in Table 4. Table 5 gives the ${ }^{13} \mathrm{CO}$ fluxes and ${ }^{12 / 13} \mathrm{CO}$ line ratios for both transitions.

\section{Molecular clouds in the outer disk of M 33}

In this article we present $\mathrm{CO}$ and even ${ }^{13} \mathrm{CO}$ detections beyond the $R_{25}$ radius of $\mathrm{M} 33$. These are the first such detections for a sub-solar metallicity galaxy. Only a few detections have been made in the outer parts of galaxies: in the Milky Way, using the rotation curve to estimate the distance (e.g. Brand et al. 1987; Digel et al. 1994), in NGC 4414 (Braine \& Herpin 2004), and in NGC 6946 (Braine et al. 2007). One of the issues is whether large quantities of molecular gas could have escaped detection in the outer disk (Pfenniger et al. 1994). In very nearby galaxies
Table 1. Positions observed in CO.

\begin{tabular}{|c|c|c|c|c|}
\hline Name & $\overline{\mathrm{RA}}$ & $\overline{\overline{D e c}}$ & $\begin{array}{c}\delta \text { RA } \\
\left({ }^{\prime \prime}\right)\end{array}$ & $\begin{array}{c}\delta \mathrm{Dec} \\
\left({ }^{\prime \prime}\right)\end{array}$ \\
\hline M33_1 & $01: 34: 17.67$ & $31: 05: 12.0$ & 0.0 & 0.0 \\
\hline \multirow{5}{*}{ M33_2 } & 01:34:06.13 & 31:03:09.7 & 0.0 & 0.0 \\
\hline & & & -20.5 & -0.0 \\
\hline & & & -0.0 & -20.5 \\
\hline & & & 20.5 & -0.0 \\
\hline & & & -0.0 & 20.5 \\
\hline \multirow[t]{3}{*}{ M33_3 } & 01:34:34.86 & $31: 00: 42.9$ & 0.0 & 0.0 \\
\hline & & & -20.5 & 0.0 \\
\hline & & & 0.0 & -20.5 \\
\hline \multirow[t]{13}{*}{ M33_4 } & 01:34:16.81 & $30: 59: 30.0$ & -10.5 & -10.5 \\
\hline & & & -10.5 & -0.0 \\
\hline & & & -10.5 & 10.5 \\
\hline & & & 0.0 & -10.5 \\
\hline & & & 0.0 & 0.0 \\
\hline & & & 0.0 & 10.5 \\
\hline & & & 10.5 & -10.5 \\
\hline & & & 10.5 & 0.0 \\
\hline & & & 10.5 & 10.5 \\
\hline & & & -21.0 & -0.0 \\
\hline & & & 21.0 & 0.0 \\
\hline & & & 0.0 & -21.0 \\
\hline & & & -0.0 & 21.0 \\
\hline \multirow[t]{5}{*}{ M33_5 } & 01:34:03.06 & $30: 58: 05.2$ & 0.0 & 0.0 \\
\hline & & & -20.5 & 0.0 \\
\hline & & & 0.0 & -20.5 \\
\hline & & & 20.5 & -0.0 \\
\hline & & & -0.0 & 20.5 \\
\hline \multirow[t]{5}{*}{ M33_7 } & $01: 34: 22.37$ & 30:57:03.1 & 0.0 & 0.0 \\
\hline & & & -20.5 & -0.0 \\
\hline & & & -0.0 & -20.5 \\
\hline & & & 20.5 & -0.0 \\
\hline & & & -0.0 & 20.5 \\
\hline \multirow[t]{5}{*}{ M33_8 } & 01:34:29.94 & $30: 57: 10.1$ & -20.5 & -0.0 \\
\hline & & & 20.5 & 0.0 \\
\hline & & & 0.0 & 20.5 \\
\hline & & & 0.0 & -20.5 \\
\hline & & & 0.0 & 0.0 \\
\hline \multirow[t]{9}{*}{ M33_9 } & 01:34:03.72 & $30: 55: 01.8$ & -20.5 & -20.5 \\
\hline & & & -20.5 & -0.0 \\
\hline & & & -20.5 & 20.5 \\
\hline & & & 20.5 & -20.5 \\
\hline & & & 20.5 & 0.0 \\
\hline & & & 20.5 & 20.5 \\
\hline & & & -0.0 & 20.5 \\
\hline & & & -0.0 & -20.5 \\
\hline & & & 0.0 & 0.0 \\
\hline \multirow[t]{2}{*}{ M33_10 } & $01: 34: 16.23$ & $30: 52: 08.4$ & 3.7 & 10.5 \\
\hline & & & 0.0 & 0.0 \\
\hline \multirow[t]{2}{*}{ M33_11 } & 01:33:59.46 & $30: 49: 12.1$ & -4.6 & -28.6 \\
\hline & & & 0.0 & 0.0 \\
\hline M33_12 & 01:34:09.30 & $30: 49: 06.1$ & 0.0 & 0.0 \\
\hline \multirow[t]{11}{*}{ M33_18 } & $01: 34: 13.31$ & $31: 10: 32.0$ & 0.0 & 0.0 \\
\hline & & & -20.5 & 0.0 \\
\hline & & & -0.0 & -20.5 \\
\hline & & & 20.5 & 0.0 \\
\hline & & & 0.0 & 20.5 \\
\hline & & & -0.0 & -10.5 \\
\hline & & & -10.5 & -0.0 \\
\hline & & & 0.0 & -41.0 \\
\hline & & & -0.0 & -61.5 \\
\hline & & & -0.0 & -82.0 \\
\hline & & & -0.0 & -102.5 \\
\hline \multirow[t]{4}{*}{ M33_20 } & 01:34:39.79 & $31: 08: 23.8$ & 0.0 & 0.0 \\
\hline & & & 30.0 & -0.0 \\
\hline & & & 60.0 & 0.0 \\
\hline & & & 90.0 & -0.0 \\
\hline
\end{tabular}


Table 1. continued.

\begin{tabular}{lcccc}
\hline \hline Name & RA & Dec & $\begin{array}{c}\delta \text { RA } \\
\left({ }^{\prime \prime}\right)\end{array}$ & $\begin{array}{c}\delta \text { Dec } \\
\left({ }^{\prime \prime}\right)\end{array}$ \\
\hline & & & 120.0 & 0.0 \\
& & & 150.0 & -0.0 \\
& & & 180.0 & 0.0 \\
& & & 210.0 & 0.0 \\
M33_22 & $01: 34: 54.09$ & $31: 13: 25.5$ & 0.0 & 0.0 \\
M33_23 & $01: 34: 45.28$ & $31: 12: 57.5$ & 0.0 & 0.0 \\
& & & 22.6 & 5.6 \\
& & & 45.2 & 11.2 \\
& & & 67.8 & 16.8 \\
& & & 90.4 & 22.4 \\
M33_24 & $01: 34: 52.39$ & $31: 08: 36.0$ & -10.5 & 0.0 \\
M33_25 & $01: 34: 22.69$ & $31: 11: 54.4$ & 0.0 & 0.0 \\
& & & -0.0 & -10.5 \\
M33_26 & $01: 34: 40.88$ & $31: 13: 40.0$ & 0.0 & 0.0 \\
M33_27 & $01: 34: 51.85$ & $31: 09: 39.6$ & 0.0 & 0.0 \\
M33_34 & $01: 34: 57.40$ & $31: 05: 13.9$ & 0.0 & 0.0 \\
\hline
\end{tabular}

Table 2. $\mathrm{CO}(1-0)$ detections.

\begin{tabular}{ccccc}
\hline Name & $\begin{array}{c}\delta \mathrm{RA} \\
\left({ }^{\prime \prime}\right)\end{array}$ & $\begin{array}{c}\delta \mathrm{Dec} \\
\left({ }^{\prime \prime}\right)\end{array}$ & $\begin{array}{c}I_{\mathrm{CO}(1-0)} \\
\left(\mathrm{K} \mathrm{km} \mathrm{s}^{-1}\right)\end{array}$ & $\begin{array}{c}\mathrm{rms} \\
(\mathrm{mK})\end{array}$ \\
\hline M33_2 & 0.0 & 0.0 & $0.20 \pm 0.03$ & 7.1 \\
M33_3 & 0.0 & 0.0 & $0.42 \pm 0.06$ & 17.5 \\
M33_4 & -10.5 & -10.5 & $1.20 \pm 0.09$ & 21.1 \\
M33_4 & -10.5 & 0.0 & $1.50 \pm 0.08$ & 19.1 \\
M33_4 & -10.5 & 10.5 & $0.99 \pm 0.13$ & 21.5 \\
M33_4 & 0.0 & -10.5 & $1.20 \pm 0.06$ & 17.7 \\
M33_4 & 0.0 & 0.0 & $1.83 \pm 0.05$ & 12.5 \\
M33_4 & 0.0 & 10.5 & $1.25 \pm 0.08$ & 16.4 \\
M33_4 & 10.5 & -10.5 & $0.64 \pm 0.06$ & 19.3 \\
M33_4 & 10.5 & 0.0 & $0.79 \pm 0.06$ & 16.9 \\
M33_4 & 10.5 & 10.5 & $0.60 \pm 0.08$ & 17.9 \\
M33_4 & -21.0 & 0.0 & $0.99 \pm 0.07$ & 17.3 \\
M33_4 & 0.0 & 21.0 & $0.94 \pm 0.06$ & 15.9 \\
M33_5 & 0.0 & 0.0 & $1.47 \pm 0.06$ & 14.9 \\
M33_5 & -20.5 & 0.0 & $0.24 \pm 0.05$ & 15.5 \\
M33_5 & 20.5 & 0.0 & $0.48 \pm 0.05$ & 16.2 \\
M33_5 & 0.0 & 20.5 & $0.42 \pm 0.07$ & 16.8 \\
M33_7 & 0.0 & 0.0 & $1.50 \pm 0.04$ & 12.5 \\
M33_7 & -20.5 & 0.0 & $0.36 \pm 0.07$ & 17.9 \\
M33_7 & 20.5 & 0.0 & $0.41 \pm 0.07$ & 16.4 \\
M33_7 & 0.0 & 20.5 & $1.04 \pm 0.06$ & 16.5 \\
M33_8 & -20.5 & 0.0 & $0.67 \pm 0.08$ & 18.3 \\
M33_8 & 0.0 & 20.5 & $0.75 \pm 0.07$ & 18.0 \\
M33_8 & 0.0 & -20.5 & $0.35 \pm 0.08$ & 16.5 \\
M33_8 & 0.0 & 0.0 & $1.44 \pm 0.04$ & 8.1 \\
M33_9 & -20.5 & -20.5 & $0.53 \pm 0.08$ & 25.0 \\
M33_9 & 20.5 & -20.5 & $0.88 \pm 0.10$ & 29.6 \\
M33_9 & 20.5 & 0.0 & $0.59 \pm 0.07$ & 20.6 \\
M33_9 & 0.0 & 20.5 & $1.25 \pm 0.08$ & 22.1 \\
M33_9 & 0.0 & -20.5 & $0.74 \pm 0.15$ & 21.8 \\
M33_9 & 0.0 & 0.0 & $2.51 \pm 0.04$ & 11.7 \\
M33_10 & 3.7 & 10.5 & $3.46 \pm 0.14$ & 28.9 \\
M33_10 & 0.0 & 0.0 & $3.55 \pm 0.08$ & 18.4 \\
M33_11 & -4.6 & -28.6 & $9.99 \pm 0.17$ & 33.5 \\
M33_11 & 0.0 & 0.0 & $9.73 \pm 0.08$ & 16.9 \\
M33_12 & 0.0 & 0.0 & $22.78 \pm 0.10$ & 18.9 \\
M33_18 & 0.0 & 0.0 & $0.52 \pm 0.06$ & 19.5 \\
M33_18 & -20.5 & 0.0 & $0.26 \pm 0.06$ & 19.8 \\
M33_18 & 0.0 & -10.5 & $0.24 \pm 0.03$ & 9.8 \\
M33_18 & -10.5 & 0.0 & $0.61 \pm 0.02$ & 7.6 \\
M33_20 & 120.0 & 0.0 & $0.26 \pm 0.03$ & 11.4 \\
M33_20 & 150.0 & 0.0 & $0.33 \pm 0.04$ & 10.2 \\
\hline & & & &
\end{tabular}

Table 3. $\mathrm{CO}(2-1)$ detections.

\begin{tabular}{lcccc}
\hline \hline Name & $\begin{array}{c}\delta \text { RA } \\
\left({ }^{\prime \prime}\right)\end{array}$ & $\begin{array}{c}\delta \text { Dec } \\
\left({ }^{\prime \prime}\right)\end{array}$ & $\begin{array}{c}I_{\mathrm{CO}(2-1)}\left(\mathrm{K} \mathrm{km} \mathrm{s}^{-1}\right) \\
(\mathrm{mK})\end{array}$ & $\begin{array}{c}\text { rms } \\
(\mathrm{mK}\end{array}$ \\
\hline M33_2 & 0.0 & 0.0 & $0.24 \pm 0.02$ & 11.6 \\
M33_3 & 0.0 & 0.0 & $0.67 \pm 0.12$ & 40.8 \\
M33_4 & 0.0 & 0.0 & $0.57 \pm 0.06$ & 31.2 \\
M33_4 & -10.5 & -10.5 & $1.04 \pm 0.11$ & 40.5 \\
M33_4 & -10.5 & 0.0 & $1.09 \pm 0.11$ & 42.4 \\
M33_4 & 0.0 & -10.5 & $0.81 \pm 0.08$ & 40.2 \\
M33_4 & 0.0 & 0.0 & $1.31 \pm 0.04$ & 19.7 \\
M33_4 & 0.0 & 10.5 & $0.56 \pm 0.10$ & 33.5 \\
M33_4 & 10.5 & 10.5 & $0.30 \pm 0.06$ & 41.0 \\
M33_4 & -21.0 & 0.0 & $0.57 \pm 0.10$ & 37.5 \\
M33_4 & 0.0 & 21.0 & $0.84 \pm 0.11$ & 41.5 \\
M33_5 & 0.0 & 0.0 & $1.38 \pm 0.09$ & 32.0 \\
M33_5 & 0.0 & 20.5 & $0.37 \pm 0.09$ & 34.9 \\
M33_7 & 0.0 & 0.0 & $1.40 \pm 0.05$ & 21.5 \\
M33_7 & 0.0 & 20.5 & $0.83 \pm 0.08$ & 34.2 \\
M33_8 & 0.0 & 20.5 & $0.97 \pm 0.09$ & 40.9 \\
M33_8 & 0.0 & 0.0 & $2.28 \pm 0.06$ & 14.8 \\
M33_9 & 0.0 & 20.5 & $0.85 \pm 0.08$ & 35.4 \\
M33_9 & 0.0 & 0.0 & $2.34 \pm 0.05$ & 19.6 \\
M33_9 & 20.5 & -20.5 & $1.09 \pm 0.09$ & 40.2 \\
M33_10 & 3.7 & 10.5 & $2.84 \pm 0.09$ & 27.1 \\
M33_10 & 0.0 & 0.0 & $2.99 \pm 0.06$ & 18.7 \\
M33_11 & -4.6 & -28.6 & $6.86 \pm 0.09$ & 25.6 \\
M33_11 & 0.0 & 0.0 & $10.72 \pm 0.07$ & 21.0 \\
M33_12 & 0.0 & 0.0 & $23.76 \pm 0.10$ & 25.7 \\
M33_18 & 0.0 & 0.0 & $0.40 \pm 0.05$ & 31.4 \\
M33_18 & -10.5 & 0.0 & $1.22 \pm 0.03$ & 17.6 \\
\hline
\end{tabular}

Table 4. Results of Gaussian fits to the detected clouds in $\mathrm{CO}(1-0)$.

\begin{tabular}{lcccc}
\hline \hline Name & $\begin{array}{c}\text { Velocity } \\
\left(\mathrm{km} \mathrm{s}^{-1}\right)\end{array}$ & $\begin{array}{c}\text { Line width } \\
\left(\mathrm{km} \mathrm{s}^{-1}\right)\end{array}$ & $\begin{array}{c}M_{\text {vir }} \\
\left(1000 M_{\odot}\right)\end{array}$ & $\begin{array}{c}M_{\text {lum }} \\
\left(1000 M_{\odot}\right)\end{array}$ \\
\hline M33_2 & $-254.1 \pm 0.5$ & $5.0 \pm 1.2$ & $125 \pm 60$ & 6 \\
M33_9 & $-256.2 \pm 0.1$ & $5.3 \pm 0.2$ & $140 \pm 9$ & 81 \\
M33_11 & $-251.2 \pm 0.1$ & $8.5 \pm 0.1$ & $361 \pm 10$ & 312 \\
M33_12 & $-248.2 \pm 0.0$ & $11.4 \pm 0.1$ & $643 \pm 11$ & 730 \\
M33_3 & $-258.7 \pm 0.4$ & $5.5 \pm 1.2$ & $150 \pm 66$ & 20 \\
M33_4 & $-269.8 \pm 0.1$ & $4.4 \pm 0.2$ & $95 \pm 7$ & 58 \\
M33_5 & $-263.0 \pm 0.1$ & $6.7 \pm 0.3$ & $221 \pm 23$ & 55 \\
M33_7 & $-263.9 \pm 0.1$ & $4.1 \pm 0.2$ & $82 \pm 9$ & 48 \\
M33_8 & $-271.6 \pm 0.2$ & $10.7 \pm 0.4$ & $567 \pm 40$ & 44 \\
M33_10 & $-267.1 \pm 0.1$ & $7.6 \pm 0.2$ & $284 \pm 17$ & 105 \\
M33_18 & $-263.3 \pm 0.1$ & $2.5 \pm 0.2$ & $31 \pm 4$ & 19 \\
\hline
\end{tabular}

Notes. Velocities and line widths, with the errors estimated by the Gaussian fitting algorithm in CLASS are provided in Cols. 2 and 3. Making the assumption that the cloud radius is equal to the beamsize, virial masses are calculated as in Braine et al. (1997a) and the errors using only the uncertainty in $\Delta V$. Masses from the $\mathrm{CO}(1-0)$ line intensity are given in Col. 5 using the line area from Table $2 \mathrm{a}$ and a "standard" galactic conversion $N\left(\mathrm{H}_{2}\right) / I_{\mathrm{CO}(1-0)}=2 \times 10^{20} \mathrm{~cm}^{-2} /\left(\mathrm{K} \mathrm{km} \mathrm{s}^{-1}\right)$. The first 4 clouds have Gaussian fits made using the $2.6 \mathrm{~km} \mathrm{~s}^{-1}$ channel width whereas the latter 7 fits have been made using data at $0.52 \mathrm{~km} \mathrm{~s}^{-1}$ channel width. Cloud 8 appears to have two velocity components, producing the broad line. Cloud 2 has a large error associated with the $\mathrm{CO}(1-0)$ observation; the Gaussian fit to the $\mathrm{CO}(2-1)$ line yields a width of $2.2 \pm 0.44 \mathrm{~km} \mathrm{~s}^{-1}$ but the $\mathrm{CO}(2-1)$ beam is much smaller and may not include all the emission.

such as M33, molecular clouds can be spatially resolved, allowing the use of the Virial theorem and isotopic lines to (roughly) assess their masses and resemblance to inner disk clouds. This is particularly interesting for small, chemically young, galaxies like M 33. 
Table 5. ${ }^{13} \mathrm{CO}$ observations.

\begin{tabular}{lccccc}
\hline \hline Name & $\begin{array}{c}I_{(1-0)} \\
\mathrm{K} \mathrm{km} \mathrm{s}^{-1}\end{array}$ & $\begin{array}{c}\sigma_{1-0} \\
\mathrm{mK}\end{array}$ & $\begin{array}{c}I_{(2-1)} \\
\mathrm{K} \mathrm{km} \mathrm{s}^{-1}\end{array}$ & $I\left(\frac{12}{13}\right)_{(1-0)}$ & $I\left(\frac{12}{13}\right)_{(2-1)}$ \\
\hline M33_4 & $0.15 \pm 0.02$ & 5.6 & $31.2 \mathrm{mK}$ & 12.2 & - \\
M33_7 & $0.14 \pm 0.02$ & 4.2 & $0.16 \pm 0.01$ & 10.7 & 8.5 \\
M33_8 & $0.13 \pm 0.02$ & 3.3 & $0.23 \pm 0.02$ & 11.2 & 10.0 \\
M33_9 & $0.16 \pm 0.01$ & 3.9 & $0.23 \pm 0.04$ & 15.7 & 10.1 \\
M33_10 & $0.29 \pm 0.02$ & 5.2 & $0.22 \pm 0.02$ & 12.2 & 13.8 \\
M33_11 & $0.77 \pm 0.02$ & 5.1 & $1.09 \pm 0.04$ & 12.6 & 9.8 \\
M33_12 & $1.78 \pm 0.02$ & 4.6 & $1.21 \pm 0.03$ & 12.8 & 19.7 \\
M33_18 & $0.07 \pm 0.01$ & 2.9 & $0.11 \pm 0.02$ & 8.9 & 11.4 \\
\hline
\end{tabular}

After the mapping of the northern part of M 33 by Gardan et al. (2007), we wanted to integrate more deeply at positions where we thought $\mathrm{CO}$ might be found, with the goal of testing whether we could predict from other means if molecular gas were present. As molecular clouds form out of the atomic gas, which dominates in the outer disk, one of the criteria was the presence of high HI column density. Dust emission is frequently linked to $\mathrm{CO}$ emission but in the outer parts of M 33 the FIR and MIR emission was often too weak to be detected by Spitzer (or earlier satellites), despite the substantial HI column densities. A series of positions was observed to a lower noise level, and in $\mathrm{CO}(1-0)$, rather than in the $\mathrm{CO}(2-1)$ mapping by Gardan et al. (2007); the coordinates are given in Table 1 and they are shown in Fig. 1 as circles. Figures 2-4 present the $\mathrm{CO}(1-0)$ and HI spectra at these positions on the $70 \mu$ m image with HI column density contours. The HI data is at $17^{\prime \prime}$ resolution from a mosaic of VLA C and D configuration fields described in Gratier et al. (2010a). These outer positions were chosen because either star formation was present from the $\mathrm{H} \alpha$ image (Greenawalt 1998; Hoopes \& Walterbos 2000) or high HI antenna temperatures (either narrow lines or high total column densities) were found in the Deul \& van der Hulst (1987) data. The FIR data was not available at the time of the observations yet the spectra show that the $\mathrm{CO}$ detections are not always at the HI or FIR/H $\alpha$ maxima although they tend to be part of larger HI structures with $\mathrm{H} \alpha$ emission. At higher sensitivity, the FIR emission would probably be detected as well. Here we show the $70 \mu \mathrm{m}$ data but the $160 \mu \mathrm{m}$ images do not show more extended emission. Figures 2-4 show the sources labelled M 33 18, 20, 22, 23, 24, 26, and 27 - all of which are north of Dec 31:05. Only deep NIR images detect the stars at these distances from the center.

Figure 5 shows the $\mathrm{CO}(1-0)$ and $\mathrm{CO}(2-1)$ spectra of the outer disk clouds M33_2, M33_3, M33_5, and the CO(1-0) spectra of the two detected positions in M33_20. Figure 6 shows the 8 clouds for which ${ }^{13} \mathrm{CO}$ emission was detected as well. Among the ${ }^{13} \mathrm{CO}$ detections is cloud M33_18, beyond the $R_{25}$ radius (see Fig. 3 as well). This is the first such detection and ${ }^{13} \mathrm{CO}$ is detected in both transitions. It is also of interest that the line widths decrease significantly from the inner regions (bottom of figure) to the outermost parts (top). The narrow lines show that we are only observing a single cloud, allowing us to try to use the Virial theorem in the next section to estimate the cloud masses. Cloud 4 (the Gardan et al. 2007, "Lonely Cloud") is very welldetected but is in an interarm region with little or no detected star formation despite the strong CO emission. Clouds 4 and 18 have been observed with the Plateau de Bure Interferometer and will be discussed at length in a dedicated article.

While there is a general link between $\mathrm{CO}$ emission and $\mathrm{HI}$ and FIR (e.g. $70 \mu \mathrm{m}$ ) emission, the CO emission of clouds 4 and 18 appears surprising even with the benefit of hindsight. The
$70 \mu \mathrm{m}$ emission, for example, is only very marginally detected at the positions of these strong CO detections. However, something must provoke the condensation of atomic gas clouds to form $\mathrm{H}_{2}$. In the case of cloud 18, the HI spectra are complicated so perhaps the $\mathrm{H}_{2}$ is the result of merging $\mathrm{HI}$ clouds (Brouillet et al. 1992; Ballesteros-Paredes et al. 1999; Hennebelle \& Pérault 1999; Heitsch et al. 2005). In the other molecular clouds in the outer regions of M 33, this seems less likely because the HI spectra are not wider or more complicated than in many places where $\mathrm{CO}$ is not found.

\section{Determining the $\mathrm{H}_{2}$ mass}

\subsection{Via the Virial theorem applied to individual clouds}

Historically, cloud masses have been estimated assuming that molecular clouds are bound under their own gravity. Galactic GMCs show a relationship between size and linewidth (Larson 1981) which is difficult to explain in other ways. The masses calculated are usually referred to as "Virial masses", twice as large as marginally gravitationally bound clouds. In addition to GMCs, diffuse clouds are also observed in the Galaxy (Polk et al. 1988) and these are probably unbound. The scales we observe in M 33 correspond to GMCs, justifying the use of the Virial method. Since the only force opposing gravity in the standard calculations is the internal motion, measured by the line width, magnetic support could increase masses further if clouds really were stable long-lived "Virialized" objects. Since the idea of virialized clouds came out, it has become clear that GMCs are not long-lived entities but rather have "lifetimes" of order $10^{7}$ years (e.g. Kawamura et al. 2009). However, the "Virial" masses have generally been found to be consistent with other means of measuring GMC masses, such as dust measurements (Sodroski et al. 1995) or gamma-ray observations (see e.g. Combes 1991). Using the expression from Dickman et al. (1986),

$$
M_{\mathrm{vir}} \approx \frac{\Delta V^{2} D}{2 \gamma G}
$$

where $\Delta V$ is the half-power velocity width and $D$ the cloud diameter $(\sqrt{4 A / \pi}$ for other shapes). The $\gamma$ parameter is principally related to the broadening of the ${ }^{12} \mathrm{CO}$ line due to its high optical depth (see Dickman et al. 1986, for details). Using this, the cloud masses $\left(M_{\text {vir }}\right)$ are given in Table 4 , along with cloud velocities, line widths, and masses calculated using a $N\left(\mathrm{H}_{2}\right) / I_{\mathrm{CO}(1-0)}=2 \times 10^{20} \mathrm{~cm}^{-2} /\left(\mathrm{K} \mathrm{km} \mathrm{s}^{-1}\right)$ factor $\left(M_{\text {lum }}\right)$.

The Virial masses given are upper limits in the sense that we have assumed that the clouds are as big as the beam, $85 \mathrm{pc}$ in diameter, whereas this is likely an overestimate of the size of a single cloud (e.g. Solomon et al. 1987). Furthermore, multiple clouds would broaden the CO lines and again cause an overestimate of the mass. However, the $\gamma=2$ value may be overestimated and is used for consistency with Braine et al. (1997a). These authors also found, through basic radiative transfer calculations and for modestly subsolar metallicites, that a low radiation field (as for the clouds in the outer disk of M 33) could counteract the deeper $\mathrm{CO}$ photo-dissociation expected with decreasing metallicity, such that the CO-emitting part of a cloud was not necessarily smaller in an outer disk environment. Nonetheless, the virial masses given here are higher (possibly due to the large assumed size) in most cases than galactic GMCs of similar line width (using $M_{\mathrm{vir}} \approx 365 \Delta V^{4} M_{\odot}$, Eq. (6a) from Solomon et al. 1987). Attributing an uncertainty is difficult; the sources of error are chiefly the size, the $\gamma$ parameter, and the 
J. Braine et al.: Molecule and Star Formation in M 33

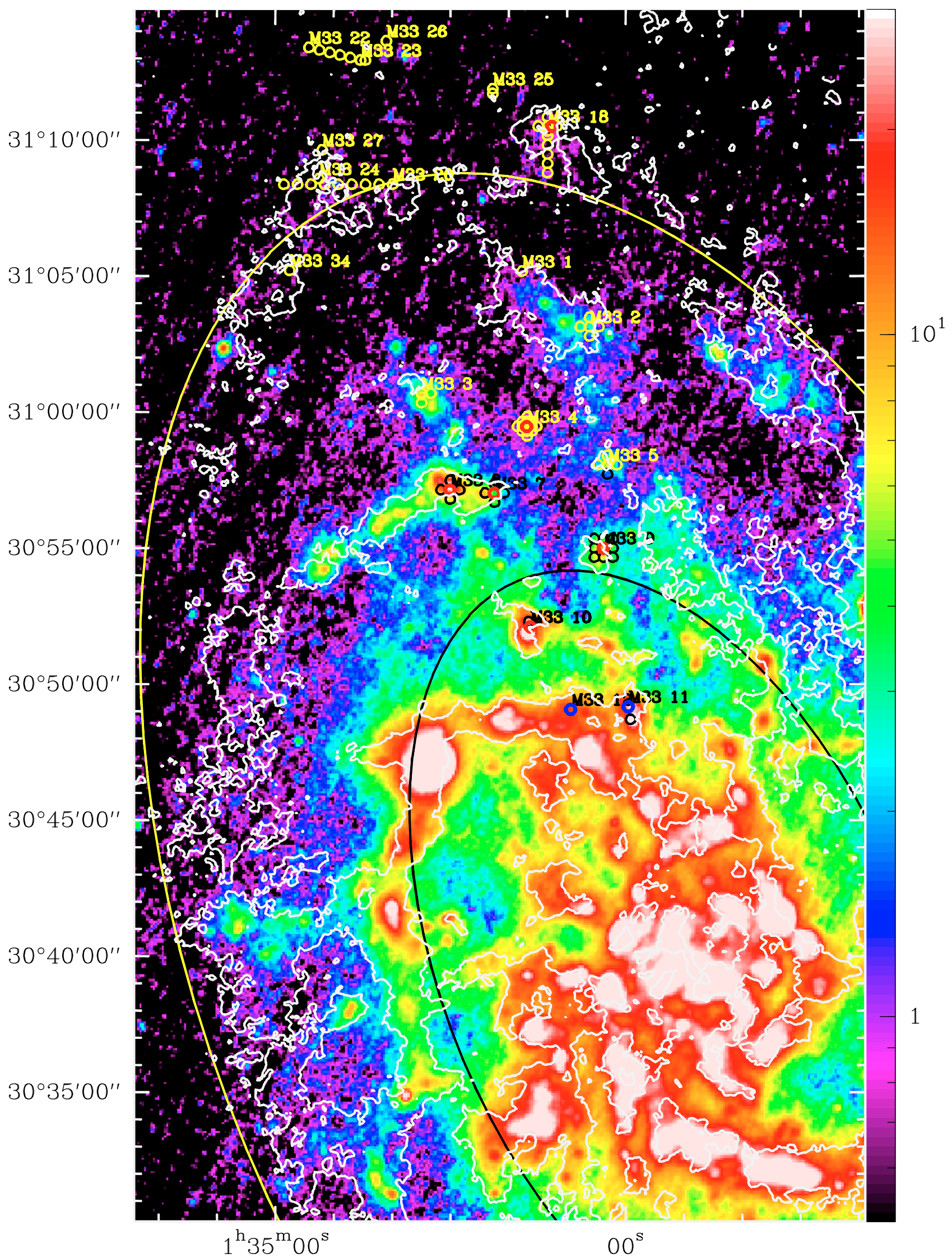

Fig. 1. $70 \mu \mathrm{m}$ image of M 33 (Tabatabaei et al. 2007) using a logarithmic scale to show the outermost features. The $R_{25}$ radius is illustrated with a yellow ellipse. The inner black ellipse indicates half the $R_{25}$ radius, where gaseous and stellar surface densities are roughly equal. The positions observed in ${ }^{12} \mathrm{CO}$ are shown in yellow in the outer parts and in black in the inner parts. Positions observed in ${ }^{13} \mathrm{CO}$ are shown in red except for the two innermost points (M33_11 and M33_12) which are shown in blue in order to be visible. All the positions observed in ${ }^{13} \mathrm{CO}$ have also been measured in ${ }^{12} \mathrm{CO}$. The white contour shows the $1.5 \times 10^{21} \mathrm{~cm}^{-2} \mathrm{HI}$ contour at $17^{\prime \prime}$ resolution from Gratier et al. (2010a). 


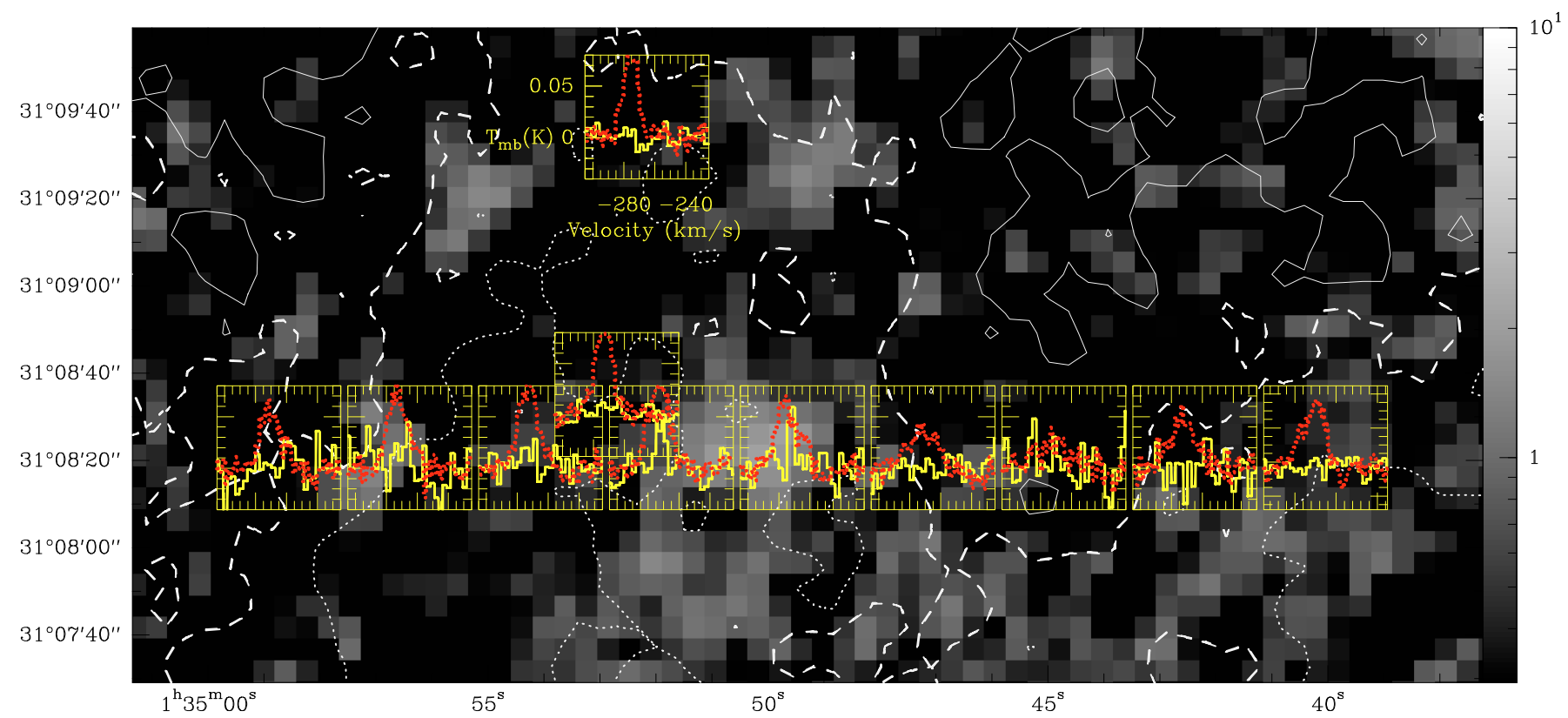

Fig. 2. $\mathrm{CO}(1-0)$ spectra (yellow) and HI spectra (red dotted) of sources M33_20, M33_24, and M33_27 on $70 \mu$ m image with HI contours at 0.8, 1.2 (dashed), and $1.6 \times 10^{21} \mathrm{~cm}^{-2}$ (dotted). The wedge shows the $70 \mu \mathrm{m}$ image scale in $\mathrm{MJy} \mathrm{sr}^{-1}$. Note that two positions along the strip (M33_20) have been detected but that they correspond to neither HI nor $70 \mu \mathrm{m}$ maxima.

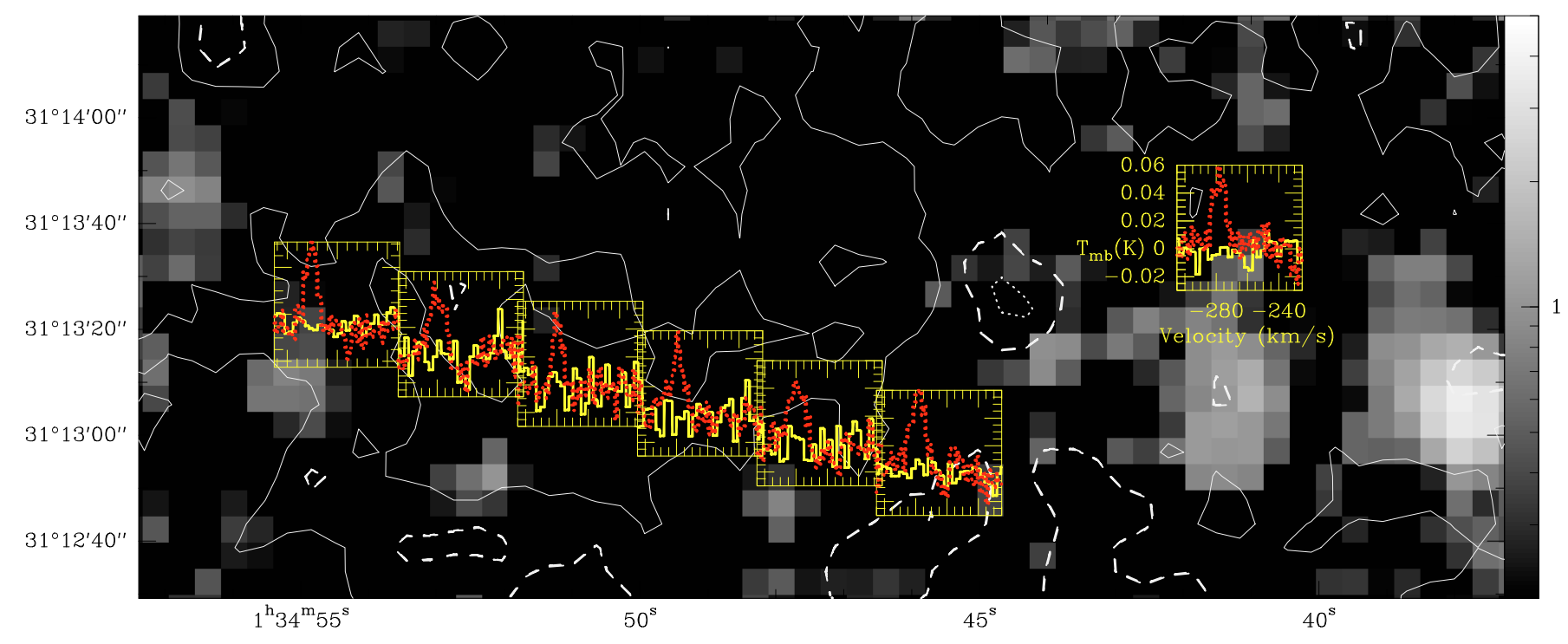

Fig. 3. $\mathrm{CO}(1-0)$ spectra (yellow) and HI spectra (red dotted) of sources M33_22, M33_23, and M33_26 on $70 \mu$ m image with HI contours at 0.8 , 1.2 (dashed), and $1.6 \times 10^{21} \mathrm{~cm}^{-2}$ (dotted). The wedge shows the $70 \mu \mathrm{m}$ image scale in $\mathrm{MJy} \mathrm{s}^{-1}$. None of the positions have been detected in CO.

hypothesis of "Virial" equilibrium (gravity alone). The size and $\gamma$ likely lead to an uncertainty of a factor 2 at most. The presence of CO-bright isolated clouds in the outer disk is evidence that they are robust, and thus gravitationally bound, structures, comforting the Virial hypothesis. If the clouds we identify were simply due to the fluctuating turbulent field then we would expect to find many weak lines for a single strong one (if indeed strong lines could be formed this way) and the Gardan et al. (2007) observations suggest this is not the case. The relatively strong ${ }^{13} \mathrm{CO}$ emission shows that the ${ }^{12} \mathrm{CO}$ line is highly optically thick, also arguing for a non-transient nature. The spectra of some clouds, particularly in the inner disk, are complicated and single-Gaussian fits lead to significant uncertainties in the linewidth and thus Virial mass. These are a minority, however, so we estimate an uncertainty of at most a factor 2 on the value given below.
With the exception of the two bright inner disk clouds, M33_11 and M33_12, which are consistent with a galactic $N\left(\mathrm{H}_{2}\right) / I_{\mathrm{CO}(1-0)}$ factor, and the very uncertain sources, the virial masses are consistent with a $N\left(\mathrm{H}_{2}\right) / I_{\mathrm{CO}(1-0)} \approx 5 \times$ $10^{20} \mathrm{~cm}^{-2} /\left(\mathrm{K} \mathrm{km} \mathrm{s}^{-1}\right)$ factor (the average for clouds $4,5,7,9$, 10 , and 18).

\subsection{From isotopic lines and ratios}

Another approach to estimating cloud masses is to measure isotopic lines assumed to be optically thin. In principle, this allows one to "count" the molecules of the optically thin species. In practice, this is quite difficult but isotopic line ratios provide a means to determine whether the GMCs are intrinsically very different from Galactic clouds. Eight of the clouds have been 


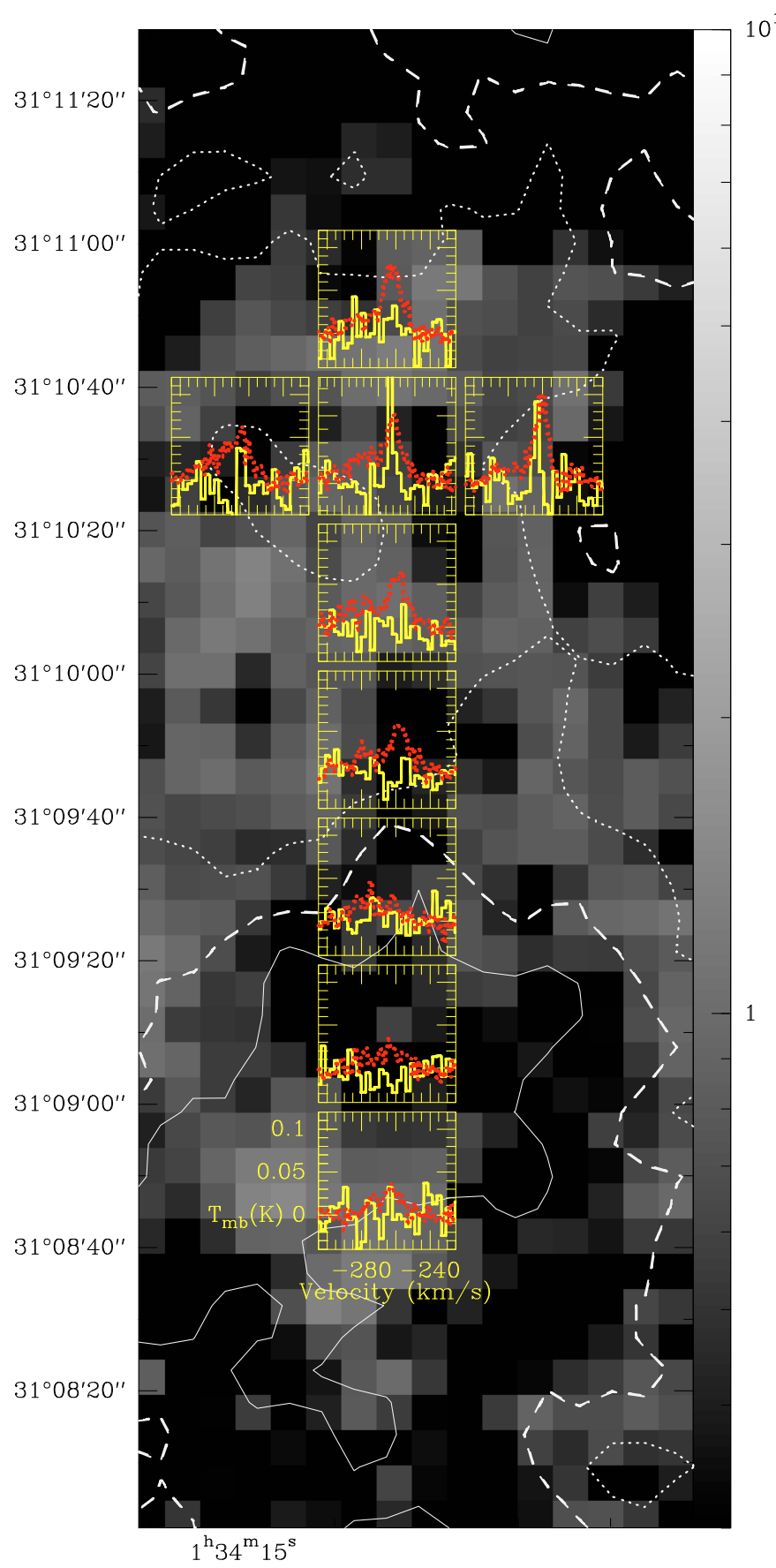

Fig. 4. $\mathrm{CO}(1-0)$ spectra (yellow) and HI spectra (red dotted) of source M33_18 and major offsets on $70 \mu \mathrm{m}$ image with HI contours at $0.8,1.2$ (dashed), and $1.6 \times 10^{21} \mathrm{~cm}^{-2}$ (dotted). The wedge shows the $70 \mu \mathrm{m}$ image scale in $\mathrm{MJy} \mathrm{sr}^{-1}$. The CO maximum is actually at the $(-10.5,0)$ position shown in Fig. 6.

observed in ${ }^{13} \mathrm{CO}$ and all of them were detected in the ${ }^{13} \mathrm{CO}(1-$ $0)$ line and all but one in ${ }^{13} \mathrm{CO}(2-1)$.

Assuming optically thin ${ }^{13} \mathrm{CO}(1-0)$ emission and Local Thermodynamic Equilibrium at a temperature $T_{\mathrm{ex}} \gg T_{\mathrm{CMB}}$, the remarkably narrow range in ${ }^{12 / 13} \mathrm{CO}$ line ratios (Table 5) yields $N\left(\mathrm{H}_{2}\right) / I_{\mathrm{CO}(1-0)}$ values within a factor 2 of $10^{20} \mathrm{~cm}^{-2} /\left(\mathrm{K} \mathrm{km} \mathrm{s}^{-1}\right)$ (see formulae 14.40 and 14.41 in Rohlfs \& Wilson 2004) for realistic excitation temperatures (assumed 10-30 K). This is true for both the ${ }^{13} \mathrm{CO}(J=1-0)$ and $J=2-1$ transitions. The calculations assumed a ${ }^{13} \mathrm{CO}$ abundance with respect to $\mathrm{H}_{2}$ of $\left(1.2 \times 10^{6}\right)^{-1}-$ lower ${ }^{13} \mathrm{CO}$ abundances will yield a higher

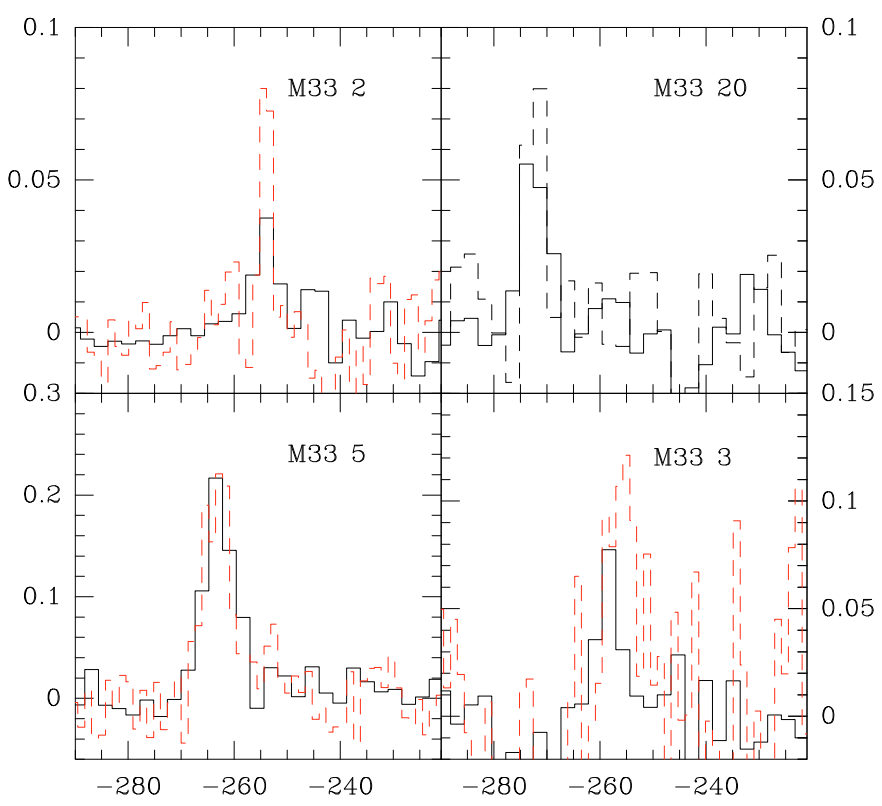

Fig. 5. Spectra of the 4 clouds where only ${ }^{12} \mathrm{CO}$ emission was detected. The $J=1 \rightarrow 0$ transition is in black and the $J=2 \rightarrow 1$ is in red (dashed). The spectra of M33_20 show the offset $(150,0)$ with a solid line and the dashed line is the $(120,0)$ offset, both in $\mathrm{CO}(1-0)$. The $x$-axis shows the velocity in $\mathrm{km} \mathrm{s}^{-1}$ and the $y$-axis the antenna temperature on the main beam scale. The order is in increasing declination from bottom to top, corresponding to increasing galactocentric distance.

$N\left(\mathrm{H}_{2}\right) / I_{\mathrm{CO}(1-0)}$ value. Of course, a fraction of the ${ }^{13} \mathrm{CO}$ is probably not optically thin and the departure from LTE will be much greater in ${ }^{13} \mathrm{CO}$ than ${ }^{12} \mathrm{CO}$ due to the inefficient radiative excitation. Furthermore, the fraction of the GMC where ${ }^{13} \mathrm{CO}$ is dissociated will be much greater than for ${ }^{12} \mathrm{CO}$. The combination of these ingredients likely explains the much lower $N\left(\mathrm{H}_{2}\right) / I_{\mathrm{CO}(1-0)}$ value obtained in this way. Low ${ }^{12 / 13} \mathrm{CO}$ line ratios lead to high $N\left(\mathrm{H}_{2}\right) / I_{\mathrm{CO}(1-0)}$ ratios.

The ${ }^{13} \mathrm{CO}$ integrated intensities and ${ }^{12 / 13} \mathrm{CO}$ line ratios for each transition are given in Table 5. The ratios are of order 10 for the $J=2-1$ transition and slightly higher for the $J=1-0$. These values are typical of (large) galaxies at large scales (Rickard \& Blitz 1985; Sage \& Isbell 1991; Paglione et al. 2001). Values for individual clouds in the Milky Way are usually lower, in the range 3-5 (Polk et al. 1988; Digel et al. 1994) but may not include all of the low column density material with significant ${ }^{12} \mathrm{CO}$ but little ${ }^{13} \mathrm{CO}$ emission. Since M 33 has a metallicity which is subsolar by a factor 2 , the ${ }^{12 / 13} \mathrm{CO}$ line ratio could be expected to be higher, as the optically thick ${ }^{12} \mathrm{CO}$ will be less affected than the ${ }^{13} \mathrm{CO}$ by the lower abundance.

The ${ }^{13} \mathrm{CO}$ spectra shown in Fig. 6 are multiplied by 10 and can be seen to lay well over the ${ }^{12} \mathrm{CO}$ spectra. The line ratios observed in M33 are thus in good agreement with the picture in which molecular clouds in M 33 are similar to their Galactic counterparts but with a lower metallicity and a $N\left(\mathrm{H}_{2}\right) / I_{\mathrm{CO}(1-0)}$ higher by about a factor two than in the Milky Way disk.

\section{Conclusions}

We present the first detections of both ${ }^{12} \mathrm{CO}$ and ${ }^{13} \mathrm{CO}$ in the extreme outer disk $\left(R>R_{25}\right)$ of a subsolar metallicity galaxy. Several clouds have been detected in our search for molecular gas in the outer disk, enabling us to try to identify the environments in which detectable quantities of $\mathrm{H}_{2}$ are found 


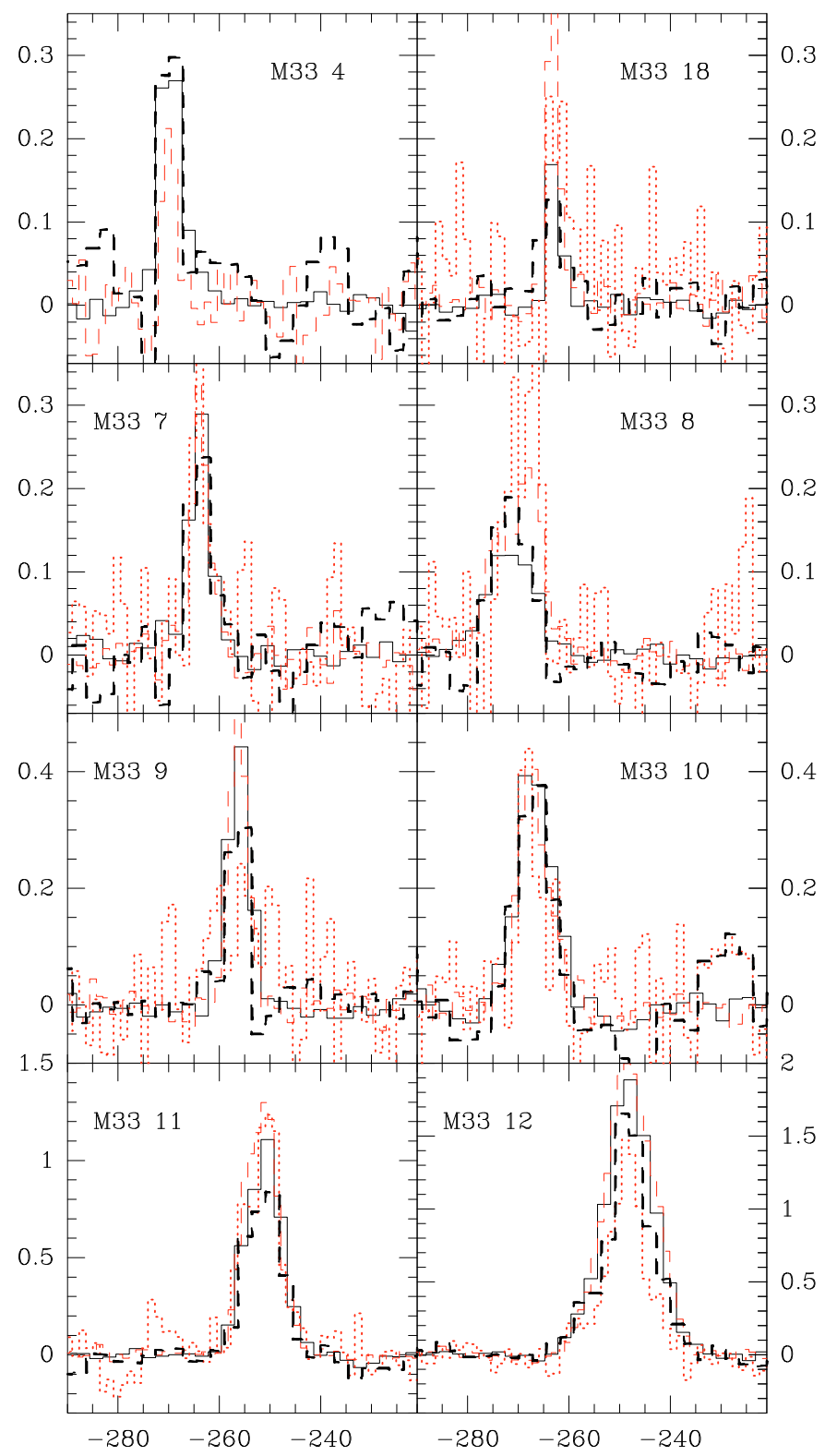

Fig. 6. Spectra of the 8 clouds where ${ }^{13} \mathrm{CO}$ emission was detected. The $J=1 \rightarrow 0$ transition is in black and the $J=2 \rightarrow 1$ is in red. The ${ }^{13} \mathrm{CO}$ antenna temperatures are multiplied by 10 . The dotted red line is the ${ }^{13} \mathrm{CO}(2-1)$ and the dashed black line is the ${ }^{13} \mathrm{CO}(1-0)$ spectrum. The $x$-axis shows the velocity in $\mathrm{km} \mathrm{s}^{-1}$ and the $y$-axis the antenna temperature on the main beam scale. The order is in increasing declination from bottom to top, corresponding to increasing galactocentric distance. Cloud 8 is most likely a blend of two clouds at different velocities (Gardan 2007) and in the spectra above there is a clear difference between the $\mathrm{CO}(1-0)$ line shape and center compared with the $(2-1)$ transition.

in the dim outer disks of spirals. The HI antenna temperature and column density and the level of star formation as traced by
FIR or $\mathrm{H} \alpha$ emission are indicators of the likelihood of detecting CO but quite imperfect because two of the strongest outer disk clouds would not have been found that way. In and of itself, the unreliability of this link is interesting.

As we are close to spatially resolving clouds, the "Virial" method is used to estimate molecular cloud masses and thus the $N\left(\mathrm{H}_{2}\right) / I_{\mathrm{CO}}$ ratio. The inner disk clouds measured are compatible with a Galactic $N\left(\mathrm{H}_{2}\right) / I_{\text {CO }}$ factor. Our estimate of $N\left(\mathrm{H}_{2}\right) / I_{\mathrm{CO}(1-0)}$ in the outer disk of $\mathrm{M} 33$ is $5 \times$ $10^{20} \mathrm{~cm}^{-2} /\left(\mathrm{K} \mathrm{km} \mathrm{s}^{-1}\right)$ with an uncertainty of at most a factor 2 . The ${ }^{12 / 13} \mathrm{CO}$ line ratios are compatible with a $N\left(\mathrm{H}_{2}\right) / I_{\mathrm{CO}(1-0)}$ factor somewhat greater than in the Milky Way molecular ring, such as the $N\left(\mathrm{H}_{2}\right) / I_{\mathrm{CO}} \sim 5 \times 10^{20} \mathrm{~cm}^{-2} /\left(\mathrm{K} \mathrm{km} \mathrm{s}^{-1}\right)$ estimated by the Virial method presented here. Adopting this conversion factor results in a lower star formation efficiency $\left(S F R / M_{\mathrm{H}_{2}}\right)$ than previously found but the SFE remains higher than the SFE in large local universe spirals (e.g. Kennicutt 1998).

\section{References}

Ballesteros-Paredes, J., Hartmann, L., \& Vázquez-Semadeni, E. 1999, ApJ, 527, 285

Braine, J., \& Herpin, F. 2004, Nature, 432, 369

Braine, J., Brouillet, N., \& Baudry, A. 1997a, A\&A, 318, 19

Braine, J., Guélin, M., Dumke, M., et al. 1997b, A\&A, 326, 963

Braine, J., Ferguson, A. M. N., Bertoldi, F., \& Wilson, C. D. 2007, ApJ, 669, L73

Brand, J., Blitz, L., Wouterloot, J. G. A., \& Kerr, F. J. 1987, A\&AS, 68, 1

Brouillet, N., Henkel, C., \& Baudry, A. 1992, A\&A, 262, L5

Combes, F. 1991, ARA\&A, 29, 195

Deul, E. R., \& van der Hulst, J. M. 1987, A\&AS, 67, 509

Dickman, R. L., Snell, R. L., \& Schloerb, F. P. 1986, ApJ, 309, 326

Digel, S., de Geus, E., \& Thaddeus, P. 1994, ApJ, 422, 92

Gardan, E. 2007, Ph.D. Thesis, Université Bordeaux 1

Gardan, E., Braine, J., Schuster, K. F., Brouillet, N., \& Sievers, A. 2007, A\&A, 473, 91

Gratier, P., Braine, J., Rodriguez-Fernandez, N. J., et al. 2010a, A\&A, 512, A68

Gratier, P., Braine, J., Rodriguez-Fernandez, N. J., et al. 2010b, A\&A, in press [arXiv: 1003.3222]

Greenawalt, B. E. 1998, Ph.D. Thesis, New Mexico State University

Heitsch, F., Burkert, A., Hartmann, L. W., Slyz, A. D., \& Devriendt, J. E. G. 2005, ApJ, 633, L113

Hennebelle, P., \& Pérault, M. 1999, A\&A, 351, 309

Hoopes, C. G., \& Walterbos, R. A. M. 2000, ApJ, 541, 597

Kawamura, A., Mizuno, Y., Minamidani, T., et al. 2009, ApJS, 184, 1

Kennicutt, R. C. 1998, ApJ, 498, 541

Larson, R. B. 1981, MNRAS, 194, 809

Leroy, A., Bolatto, A., Walter, F., \& Blitz, L. 2006, ApJ, 643, 825

Magrini, L., Stanghellini, L., \& Villaver, E. 2009, ApJ, 696, 729

Paglione, T. A. D., Wall, W. F., Young, J. S., et al. 2001, ApJS, 135, 183

Paturel, G., Petit, C., Prugniel, P., et al. 2003, A\&A, 412, 45

Pfenniger, D., Combes, F., \& Martinet, L. 1994, A\&A, 285, 79

Polk, K. S., Knapp, G. R., Stark, A. A., \& Wilson, R. W. 1988, ApJ, 332, 432

Rickard, L. J., \& Blitz, L. 1985, ApJ, 292, L57

Rohlfs, K., \& Wilson, T. L. 2004, Tools of radio astronomy, ed. K. Rohlfs, \& T. L. Wilson

Rosolowsky, E., \& Simon, J. D. 2008, ApJ, 675, 1213

Rubio, M., Garay, G., Montani, J., \& Thaddeus, P. 1991, ApJ, 368, 173

Sage, L. J., \& Isbell, D. W. 1991, A\&A, 247, 320

Sodroski, T. J., Odegard, N., Dwek, E., et al. 1995, ApJ, 452, 262

Solomon, P. M., Rivolo, A. R., Barrett, J., \& Yahil, A. 1987, ApJ, 319, 730

Tabatabaei, F. S., Beck, R., Krause, M., et al. 2007, A\&A, 466, 509 